\title{
Energy efficient DBA algorithms for TWDM-PONs
}

\author{
Abhishek Dixit, Bart Lannoo, Didier Colle, Mario Pickavet, and Piet Demeester \\ Dep. of Information Technology, Ghent University - iMinds, Gaston Crommenlaan 8/201, B-9050 Gent, \\ Belgium \\ Tel: +32933149 77, Fax: +3293314899, e-mail: abhishek.dixit@intec.ugent.be
}

\begin{abstract}
Energy efficiency is of a vital significance in the design of next generation time and wavelength division multiplexed passive optical networks (TWDM-PONs). In this paper, we first review strategies to save energy in TWDM-PONs using the state-of-the-art dynamic bandwidth allocation (DBA) algorithms. The DBA algorithms should not only minimize energy consumption but should impose a minimal penalty on delay performance. In this context, mainly two DBA design paradigms can be exploited: offline and online. After reviewing the performance of various design paradigms, we propose an optimal algorithm, which minimizes the energy consumption at both the OLT and the ONUs, by combining the energy efficiency due to sleep modes and the load dependent use of transceivers at the OLT. Due to this, the average energy consumption is reduced to $31 \%$.
\end{abstract}

Keywords: Dynamic Bandwidth Allocation, Passive Optical Networks, and TWDM-PON.

\section{INTRODUCTION}

The incessant growth of bandwidth intensive applications like high-definition Internet video, file sharing and video conferencing requires a parallel growth in bandwidth capacities, which can be achieved by bringing an optical fibre closer to an end user, leading to the fibre-to-the-home (FTTH) technology. A promising and widely adopted FTTH technology is a passive optical network (PON). The PON mostly has a tree topology and consists of an optical line terminal (OLT) in a central office, passive splitters/combiners in a remote node $(\mathrm{RN})$ and a number of optical network units (ONU) at a user side. The PON technology can be implemented in a variety of ways, and time and wavelength division multiplexed PON (TWDM-PON) has been adopted as the primary candidate of the next generation PON solution [1]. TWDM-PON combines the expansion in capacity deposited by WDM with the inherent resource granularity of a TDM PON. TWDM-PON requires a dynamic bandwidth allocation (DBA) algorithm that dynamically assigns wavelengths and time slots to ONUs. The primary objective of a DBA algorithm is to assure a high quality of service (QoS) [2]. Together with the objective of ensuring a high QoS, the DBA algorithms should also minimize the energy consumption of TWDMPONs, as current access networks (including customer's premises equipment) consumes about $80 \%$ of the energy consumed in the Internet. In this paper, we focus on the energy efficient DBA algorithm for TWDMPONs.

A typical configuration of TWDM-PON has been shown in Figure 1. We assume four wavelengths at the OLT with an upstream line rate of $2.5 \mathrm{~Gb} / \mathrm{s}$ and a downstream line rate of $10 \mathrm{~Gb} / \mathrm{s}$, and a split of 1:512. Further, we assume a power splitter at the RN.

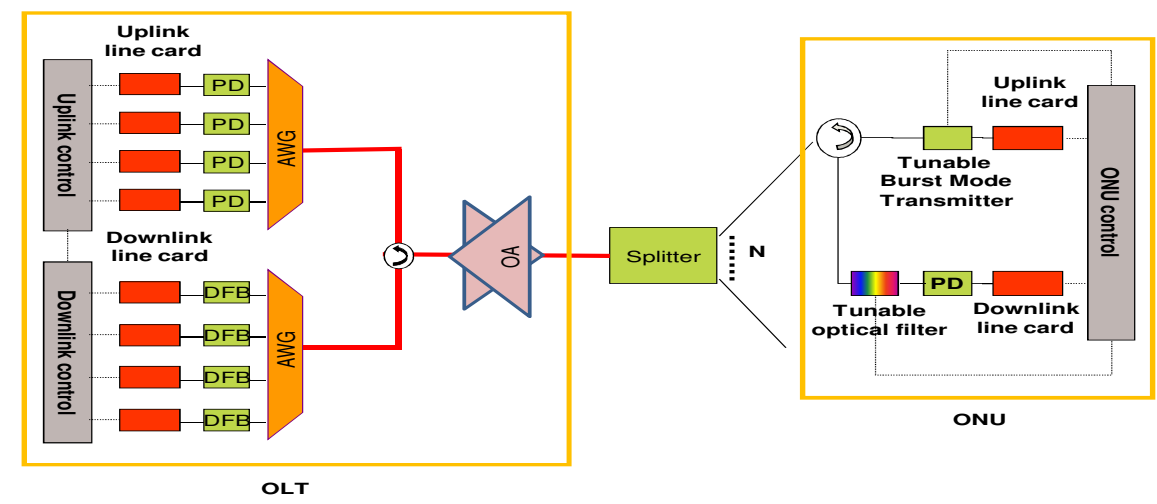

Figure 1: Architecture of a stacked TWDM-PON. Abbreviations used in the figure: DFB=distributed feedback laser, PD =photodiodes, AWG = arrayed waveguide grating, OA = Optical amplifiers

\section{Energy savings in TWDM-PON}

TWDM-PON opens up interesting vistas to save energy, both at OLTs and ONUs. The wavelengths at the OLT can be used at a high utilization by turning off idle ones, and ONUs can exploit sleep modes due to a bursty and slotted transmission. 
The energy consumption at the OLT could be reduced by using transceivers at a high utilization, leading to higher aggregation levels at the OLT and minimizing traffic burst. This can be accomplished in many ways. One approach is to use a high sharing at the RN. The second approach is to share a transceiver among many PON segments at the OLT. This does not enforce increasing split ratios in the RNs. The third approach can be applied to multi-wavelength PON systems, such as TWDM-PON, in which the number of transceivers used could be made dependent on the network load. All these approaches have significant potentials to reduce energy consumption. In this paper, we will focus on the third approach, as it involves having a proper DBA algorithm whereas the other two approaches involve designing TWDM-PON architectures.

On the other hand, the ONUs consume the major portion $(90 \%)$ of the energy consumed in optical access networks. Thus, several low power modes have been actively considered at the ONUs, e.g., sleep and doze modes [3]. In low-power modes, according to the traffic load and or at some specific conditions, the ONU's functionality that is not required is powered down. One of the types of a low-power mode is sleep mode where most of the ONU architecture is powered down when it has no traffic to receive or send.

\section{DBA approaches for TWDM-PON}

The DBAs can be approached by using either separate time and wavelength scheduling (STWS) algorithms or joint time and wavelength scheduling (JTWS) algorithms [4]. STWS algorithms decouple the wavelength assignment from time slot allocation and thus are less complex. JTWS algorithms are more complex but efficient and scalable approach.

STWA algorithms present simple solutions. The wavelength assignment can either remain static or vary with load. The static wavelength assignment, however, is not able to attain goods of statistical multiplexing. Hence, a periodic load dependent grouping of ONUs on wavelengths has been proposed in [5] to adapt to bursty traffic. The number of wavelengths can also be changed according to the load to procure energy efficiency. Thus, we proposed hybrid sleep mode aware (HSMA) algorithm in [5] to attain significant energy efficiency in TWDM-PONs.

In JTWS algorithms, the problem of wavelength assignment is tackled by using the first available wavelength (FAW) [4]. The channel availability is decided based on "horizon approach" in which each channel is considered as busy until the end of the last scheduled reservation. If no channel is free, then the wavelength with the earliest finishing last transmission (EFT) is selected and the grant for the new reservation is delayed accordingly. Another variation of FAW is Latest Finish Time (LFT) and detaining latest finish time (DLFT) [6]. To improve the channel utilization of horizon-based algorithms, the void filling approach has been proposed [7]. The OLT tracks voids (unused transmission slot) in all upstream wavelengths and schedules the transmission for an ONU within the first void that is greater than the granted upstream data and the accompanying report message. The complexity of these algorithms is higher and is dependent on the number of voids.

However, these algorithms allow unlimited wavelength switching. As the tuning time of the ONUs is non-negligible, these JTWS algorithms do not achieve acceptable performance even for tuning times in the order of microseconds. Thus, the wavelength assignment algorithm must consider wavelength switching as an important design criterion. This new design dimension can be further extended over EFT/LFT algorithms. In paper [8], we proposed EFT with optimal switching (EFT-OS) algorithm that adapts the wavelength switching to the laser tuning times, and thus obtains a high performance.

\section{Optimal design choice: STWA-OS vs. EFT-OS}

We first compare EFT-OS with the STWA approach (referred here as STWA-OS). For the details about EFT-OS, we refer the readers to [8]. In STWA-OS, we use a separate time and wavelength-scheduling algorithm; the algorithms are disjoint in upstream and downstream direction. After a fixed time T, the ONUs are regrouped on the wavelengths to maintain evenly loaded wavelengths, while being fair to the ONUs. More details of the ONU grouping will follow in section 4.1.1. STWA-OS will obviously be less complex than EFTOS and as the wavelength and time allocation is decoupled in them, they can be complemented easily with the energy efficient protocols of TDMA-PONs. However, STWA-OS will impose a higher delay penalty. In Figure 2, we compare STWA-OS with EFT-OS to show that STWA-OS can even compete with EFT-OS in the delay performance. For these results, $\mathrm{T}$ is chosen as $10 \mathrm{~ms}$. These results motivated us to use STWA-OS algorithm to accrue energy efficiency while maintaining a low complexity and a minimal delay penalty.

In the following sections, we discuss the hybrid sleep mode awareness (HSMA) algorithm, which is a variant of STWA-OS, proposed for accruing energy efficiency.

\subsection{Hybrid Sleep Mode Awareness Algorithm:}

HSMA adopts two phase scheduling: wavelength minimization and assignment (WMA) and time slot assignment (TSA). WMA tackles variability in traffic over a period, whereas TSA distributes bandwidth among ONUs on a per cycle basis. 


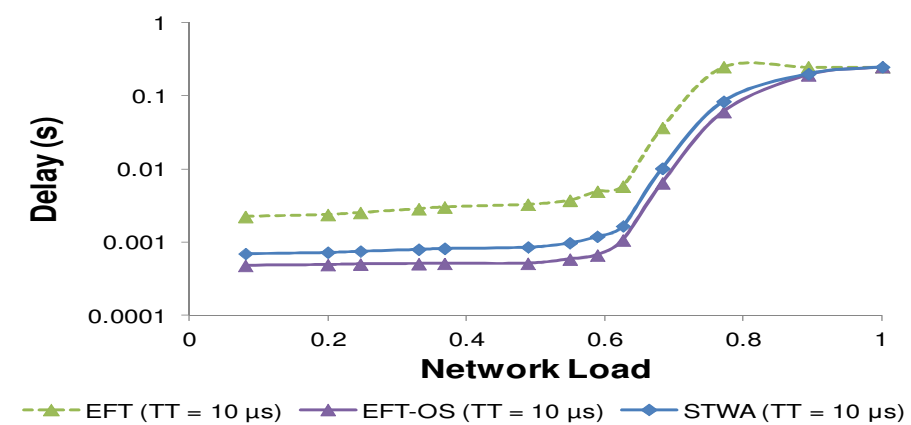

Figure 2: Comparison of EFT-OS with STWA-OS

\subsubsection{WMA:}

In WMA, the number of wavelengths and the users on a wavelength are determined after a fixed time $\mathrm{T}$. $\mathrm{T}$ can be fixed based on the operator's choice and the variability in the traffic use. If $\mathrm{T}$ is large (12 hours), then WMA is on a day-night basis, and a small $\mathrm{T}(2 \mathrm{~ms})$ tackles a more rapid variation in traffic. Note that a smaller $\mathrm{T}$ follows traffic more precisely and will harness better energy efficiency gains. Conversely, it induces penalties of large tuning times (TTs) that an ONU suffers because of hopping on to a different wavelength. Thus, it is important to ensure the fair selection of the ONUs that will hop on during a next frame (or WMA).

We use the number of wavelengths $\left(N_{w}\right)$ according to the load as $N_{w}=\left\lceil\sum_{i=1}^{N} B_{i} / T \cdot \lambda_{d}\right\rceil$, where $B_{i}$ is the sum of requested data (in bits) of ONU $i$ over a period of T, $N$ is the number of ONUs and $\lambda_{d}$ is the data rate (bits/second) of each wavelength. After deciding the number of wavelengths, another challenge is the ONUs' assignment on wavelengths that maintains evenly loaded wavelengths, while being fair to the ONUs. The ONUs are assigned on the wavelengths in a round robin manner. In a time frame $n$, the round is initiated by the ONU with id $n$ modulo $N$ (Figure 3). This is done to ensure uniform distribution of wavelength switching (or tuning times) among ONUs. An ONU is assigned to a wavelength if the normalized load of the ONU is smaller than twice the remaining capacity (where the remaining capacity is the difference between the normalized $\sum_{i=1}^{N} B_{i} / T \cdot \lambda_{d} \cdot N_{w}$

and the already assigned capacity) on the wavelength; otherwise it is deferred to the subsequent wavelength. After assignment of ONUs on a wavelength, the normalized load of other (remaining) wavelengths is updated according to the under- or over-utilization of the wavelength.

Figure 3 shows that in a frame $n$, four wavelengths are used, and the round starts with ONU 1 . In the next frame, due to a lower load, one wavelength is switched off, and the ONU assignment starts with ONU 2. Note that using a minimal number of wavelengths still satisfying the requirements of all ONUs is a bin-packing problem, which ideally requires complex heuristics (NP-hard) to optimally assign ONUs to wavelengths. In addition, the heuristics may cause some ONUs (lightly loaded) to switch more than the others causing fairness problems. Our proposed algorithm maintains simplicity and fairness; however, it may lead to more loaded wavelengths increasing delays.

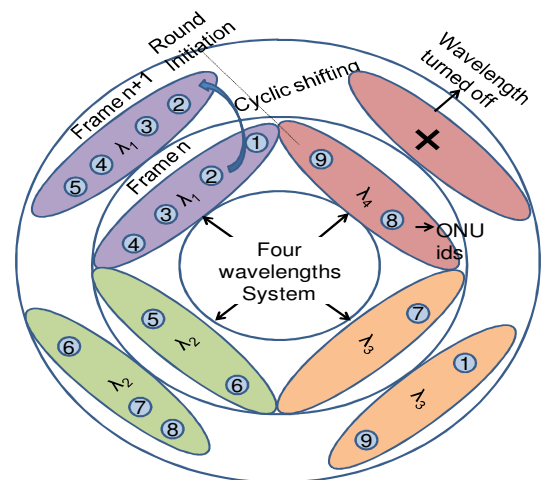

Figure 3: Wavelength assignment and grouping in the proposed algorithm.

\subsubsection{TSA:}

Within a WMA frame, the various ONUs' groups (the ONUs on a same wavelength) are assigned TDMA cycles using the SMA with up- and downstream centric (UDC) algorithm [2]. SMA-UDC proposes to 
transmit the up- and downstream traffic of an ONU at the same time for maximal energy efficiency [2] (sleep period). This entails that the ONUs be grouped identically in the up- and downstream direction: segmenting TWDM-PON into logical TDMA PONs, where a group of ONUs uses the same up- and downstream wavelength. However, due to a different load profile, the grouping of ONUs (according to WMA methods) in up- and downstream direction may be different. As a result, whichever grouping (based on down- or upstream) induces a lower peak-normalized load on a wavelength is chosen for both the directions. Note that a contrary approach can be to select the grouping according to higher wavelength utilizations: reaping higher energy efficiency at the risk of enlarging delays.

Another challenge is to maintain the TDMA cycle assignments within a WMA frame. Given the varying lengths of TDMA cycles and a fixed T (Figure 4), this is not guaranteed per se. Unchecked TDMA cycles may cross the time epoch of wavelength assignments (Figure 4), and may lead to problems of duplicated scheduling (DuS) as an ONU may get assigned to two wavelengths at the same time: one in the cycle (C) of the previous frame, and the other in the cycle of a new frame. For example, ONU 4 may be scheduled in two wavelengths at the same time (Figure 4). To solve this problem, we introduce adjusting cycles (AdC). The AdC is referred to as the last cycle in a WMA frame. Whenever, the remaining scheduling length $R_{l}$ (i.e., the difference of the total length $\mathrm{T}$ and the sum of the lengths of the previously scheduled cycles) of the WMA frame becomes shorter than the maximum cycle length $C_{\max }$, the OLT distributes $R_{l}$ among all ONUs in the ratio of their requested windows (REPORTs). Should the REPORT from an ONU not arrive at the time of the start of an AdC, the previous requested window is used (exploiting the fact that traffic is bursty and traffic requests from an ONU repeat for some cycles). In addition, in the AdC, the ONUs are informed of their next grouping and the upand downstream wavelengths they need to use.

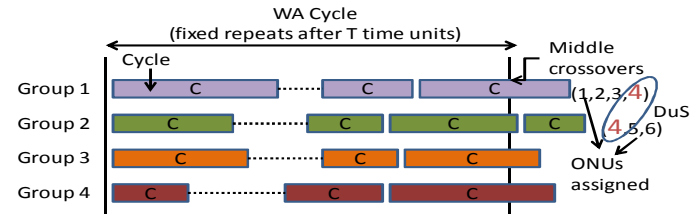

(a)

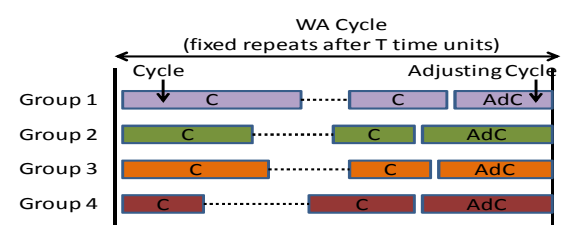

(b)

\section{Simulation Results}

In OPNET, we study the performance of HSMA by conducting a simulation of a TWDM-PON with 512 ONUs, and four wavelengths in each direction. We assumed a maximum ONU load of $100 \mathrm{Mbps}$, downstream and upstream line rate of $10 \mathrm{Gbps}$ and $2.5 \mathrm{Gbps}$, maximum OLT to ONU distance of $20 \mathrm{~km}$, maximum cycle time of $2 \mathrm{~ms}$, ONU buffer of $1 \mathrm{MB}$, T as $0.1 \mathrm{~s}$, TT of ONUs as $1 \mathrm{~ms}$, ONU power as $80 \%$ of the total (OLT+ONU) power, power in sleep mode as $15 \%$ of the active ONU power, and guard time between adjacent ONU slots as $1 \mu \mathrm{s}$. We generated traffic as in [2]. The up- and downstream normalized load is considered to vary symmetrically. In results, only upstream delay (the highest of the two) is plotted.

The energy consumption and capacity usage are shown in Figure 5. The energy consumption is reduced to an average of $31 \%$ compared to the case when there is no saving at the OLT or ONUs. At very small loads, the sleep mode periods are worse as cycle times are short, leading to frequent ONU wake ups.

We compare the delay performance of HSMA with EFT, with and without WM (wavelength minimization). We use all 4 wavelengths for "without WM" scenarios. Further, note that, in EFT, ONU grouping is not required as the users are not assigned on a wavelength per frame but per cycle. In addition, EFT-WM also suffers from middle crossovers and thus AdC is used for them. Delay performance is shown in Figure 6a. The delay oscillates because the normalized load oscillates due to the addition and deletion of wavelengths. For example, when network load is 0.2 , only one wavelength is used and the normalized load is 0.8 . However, as soon as the load crosses 0.25 , most of the time 2 wavelengths are used, leading to a normalized load of 0.5 . Hence, though network load always increases, normalized load oscillates, leading to an oscillating delay performance. Moreover, the delay performance of HSMA without WM and with WM is improved compared to EFT counterparts due to an excessive wavelength switching in EFT.

Lastly, the delay performance is shown with the variation in T and TT at the normalized load of 0.4 (Figure 6b). As TT increases, delay increases. However, increasing $\mathrm{T}$ has two different effects. First, as T increases, degradation due to TTs minimizes and secondly the network response to burstiness of traffic decreases. Due to these two counteracting effects, the optimal value of $\mathrm{T}$ is found as $0.1 \mathrm{~s}$. 


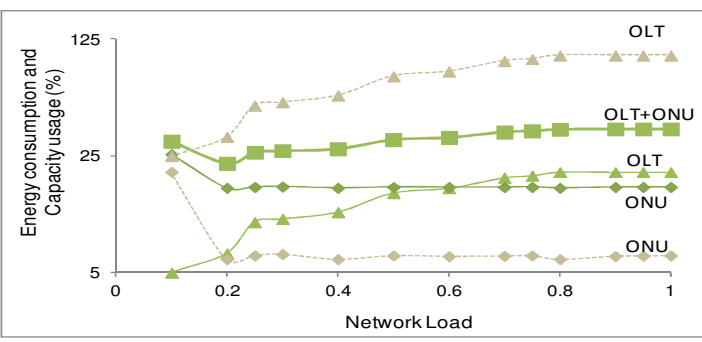

Figure 5: Energy consumption (solid lines) and capacity usage (dashed lines) in HSMA

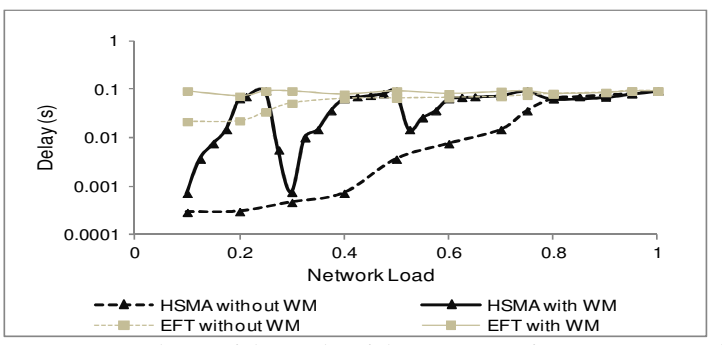

(a) Delay with and without WM in HSMA and EFT

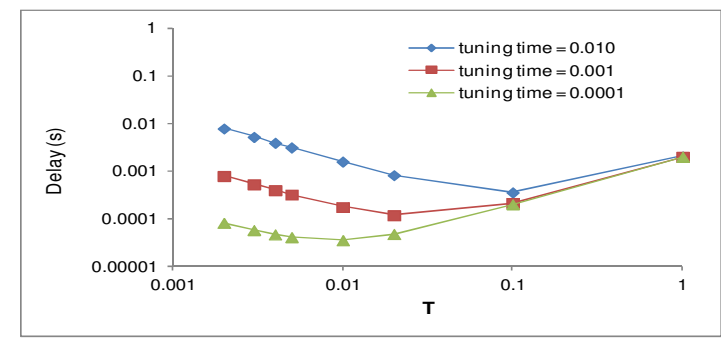

(b) Delay with a variation in TT and $\mathrm{T}$.

Figure 6: The delay performance of HSMA

\section{Conclusions}

We proposed the HSMA algorithm, which combines the energy efficiency due to sleep modes and the load dependent use of transceivers at the OLT. Due to this, the average energy consumption is reduced to $31 \%$. In addition, the delay is reduced in HSMA compared to conventional algorithms like EFT.

\section{REFERENCES}

[1] Y. Luo et al., "Time and wavelength division multiplexed passive optical network (TWDM-PON) for next generation PON stage 2 (NG-PON2)," IEEE/OSA Journal of Lightwave Technology, vol. 31, no. 4, pp. 587-593, 2013.

[2] A. Dixit et al., "Dynamic Bandwidth Allocation with SLA Awareness for QoS in Ethernet Passive Optical Networks," Journal of Optical Communications and Networking, vol. 5, no. 3, March 2013.

[3] A. Dixit et al., "ONU power saving modes in next generation optical access networks: progress, efficiency and challenges," Optics Express, vol. 20, no. 26, pp. B52-B63, 2012.

[4] K. Kanonakis, and I. Tomkos, "Improving the efficiency of online upstream scheduling and wavelength assignment in hybrid WDM/TDMA EPON networks," IEEE Journal on Selected Areas in Communications, vol. 28, no. 6, pp. 838-848, 2010.

[5] A. Dixit et al., "Novel DBA algorithm for Energy Efficiency in TWDM-PONs," in European Conference on Optical Communications (ECOC), London, U.K., 2013.

[6] K. Kanonakis and I. Tomkos, "Detaining online upstream scheduling in WDM/TDMA EPONs," in ECOC, Torino, Italy, 2010.

[7] K. Kanonakis and I. Tomkos, "Online upstream scheduling and wavelength assignment algorithms for WDM-EPON networks," in ECOC, Vienna, Austria, 2009.

[8] A. Dixit et al., "Dynamic Bandwidth Allocation with Optimal Wavelength Switching in TWDM-PONs," in International Conference on Transparent Optical Network (ICTON), Cartagena, Spain, 2013. 